"NOTICE: this is the author's version of a work that was accepted for publication in Ultramicroscopy. Changes resulting from the publishing process, such as peer review, editing, corrections, structural formatting, and other quality control mechanisms may not be reflected in this document. Changes may have been made to this work since it was submitted for publication. A definitive version was subsequently published in Ultramicroscopy, Volume 138, March 2014, Pages 46-56, DOI 10.1016/j.ultramic.2013.11.007" 


\title{
Optimized imaging using non-rigid registration
}

\author{
Benjamin Berkels ${ }^{\mathrm{a}, 1}$, Peter Binev ${ }^{\mathrm{a}, \mathrm{b}}$, Douglas A. Blom ${ }^{\mathrm{c}, *}$, Wolfgang Dahmen ${ }^{\mathrm{a}, \mathrm{d}}$, Robert C. Sharpley ${ }^{\mathrm{a}, \mathrm{b}}$, \\ Thomas $\operatorname{Vogt}^{\mathrm{a}, \mathrm{c}, \mathrm{e}}$ \\ ${ }^{a}$ Interdisciplinary Mathematics Institute, 1523 Greene Street, University of South Carolina, Columbia, SC 29208, USA \\ ${ }^{b}$ Department of Mathematics, 1523 Greene Street, University of South Carolina, Columbia, SC 29208, USA \\ ${ }^{c}$ NanoCenter, 1212 Greene Street, University of South Carolina, Columbia, SC 29208, USA \\ ${ }^{d}$ Institut für Geometrie und Praktische Mathematik, RWTH Aachen, Templergraben 55, 52056 Aachen, Germany \\ ${ }^{e}$ Department of Chemistry and Biochemistry, 631 Sumter Street, University of South Carolina, Columbia, SC 29208, USA
}

\begin{abstract}
The extraordinary improvements of modern imaging devices offer access to data with unprecedented information content. However, widely used image processing methodologies fall far short of exploiting the full breadth of information offered by numerous types of scanning probe, optical, and electron microscopies. In many applications, it is necessary to keep measurement intensities below a desired threshold. We propose a methodology for extracting an increased level of information by processing a series of data sets suffering, in particular, from high degree of spatial uncertainty caused by complex multiscale motion during the acquisition process. An important role is played by a nonrigid pixel-wise registration method that can cope with low signal-to-noise ratios. This is accompanied by formulating objective quality measures which replace human intervention and visual inspection in the processing chain. Scanning transmission electron microscopy of siliceous zeolite material exhibits the above-mentioned obstructions and therefore serves as orientation and a test of our procedures.
\end{abstract}

Keywords: non-rigid registration, Si-Y zeolite, diffusion registration, IQ-factor, HAADF-STEM

\section{Introduction}

"Learning from data" has become an indispensable pillar of science of ever increasing importance. Data acquisition can be broadly broken down into two categories, parallel acquisition and serial acquisition. In the case of serial acquisition when the change of signal between data points encodes the information of primary interest (e.g. images acquired one pixel at a time) extraction of the information from the raw data is difficult to achieve. Obstacles include low signal-to-noise ratios, possible changes of the observed object due to the observation process, and - perhaps commonly less acknowledged - uncertainties in the positioning of the observations. As a consequence, the various stages of data processing still often involve a high degree of subjective human intervention. This is particularly true,

${ }^{*}$ Correspondence to: University of South Carolina, NanoCenter, 1212 Greene St., Columbia, SC 29208, USA

Tel:+1-803-777-2886;

Fax:+1-803-777-8908;

Email addresses: berkels@aices.rwth-aachen.de

(Benjamin Berkels), binev@math.sc.edu (Peter Binev),

doug.blom@sc.edu (Douglas A. Blom),

dahmen@igpm.rwth-aachen.de (Wolfgang Dahmen),

rcsharpley@gmail.com (Robert C. Sharpley),

tvogt@mailbox.sc.edu (Thomas Vogt)

${ }^{1}$ Present Address: Aachen Institute for Advanced Study in Computational Engineering Science (AICES), RWTH Aachen University, Schinkelstr. 2, 52062 Aachen, Germany because standard methods cannot handle highly complex multiscale motion of the observed object. A representative example, providing the main orientation for this work, is scanning transmission electron microscopy (STEM). We are convinced though that the proposed methodology is scale-invariant and therefore relevant for a much wider scope of application.

The tremendous instrumental advances in STEM technology open new perspectives in understanding nanoscale properties of materials. However, in particular when dealing with beam sensitive materials, a full exploitation of this technology faces serious obstructions. The acquisition of the images takes time during which both material damage as well as environmental disturbances can build up. A major consequence is a significant - relative to the scale under consideration - highly complex motion intertwined with specimen distortion.

In response we propose a new data assimilation strategy that rests on the following two constituents:

i) replacing single frame high-dose data acquisition by taking multiple low-dose, necessarily noisy, frames;

ii) properly synthesizing the information from such series of frames by a novel cascading registration methodology.

A few comments on the basic ingredients are needed. Taking a single high-dose frame may in principle feature a higher signal-to-noise ratio but typically increases the possibility of irreparable beam damage, prevents one from accurately tracking the combination of local and global mo- 
tion during the scanning process, and possibly introduces additional unwanted physical artifacts. We use STEM imaging of a water-containing siliceous zeolite Y (Si-Y) as a representative guiding example, since it poses many of the obstacles which occur in practice when determining the structure and function of important classes of materials. In particular, Si-Y mimicks biological matter by rapidly deteriorating when exposed to high energy electron beams. Furthermore, the registration task for Si-Y poses difficult hurdles for registration which are often encountered in imaging materials, including a high degree of symmetry and low variety of structures in the presence of low signal-to-noise samples.

The core constituent i) above, i.e. taking several lowdose short-exposure frames of measurements from the same physical locations of the specimen, allows us instead to better resolve the complex motion and nonstationary distortions. We demonstrate how the information from multiple frames can be used to offset such distortions as those described.

A critical ingredient of core constituent ii) is to align the series of frames. Extracting improved information from series of low quality images is a well-known concept, usually termed super resolution in the imaging community. It rests in an essential way on the ability of accurately tracking inter-frame motion, typically using feature extraction. However, the low signal-to-noise ratios prohibit accurate feature extraction. Moreover, the physical nature of the motion encountered in series of STEM images is of a highly complex nature that can therefore not be treated satisfactorily by standard techniques. The extreme sensitivity of STEM to the measuring environment gives rise to motion which appears to exhibit at least three scales 1: jitter on the atomic level, contortion on the unit cell level, and a macro drift. As explained later in more detail, short time exposures provide the opportunity for a sufficiently refined temporal discretization. Furthermore, being able to generate highly accurate pixel to pixel mappings, in contrast to fiducial-based registration in imaging, seems to be the only hope to avoid significant blurring at the image assimilation stage.

The few existing approaches in the STEM context resort to manual rigid alignment (see e.g. in tomography [2] and single particle analysis [3]). Although in our experiments, from a subjective point of view, rigid alignment appears to yield good results, they are, however, as is typical, confined to a relatively small image portion that is implicitly used as a feature substitute. First, this introduces a highly subjective and therefore non-reproducible component into the data processing chain. Second, it wastes and even obscures information about the observed object carried by large parts of the image series. In particular, in STEM there is often high interest in determining possible irregularities in the atomic structure outside the small registration zone.

In order to be able to make full use of the information carried by the frames and, in particular, to be able to reliably detect material imperfections away from the subjective reference region we propose a powerful multilevel non-rigid registration method accurately linking the information carried by many sequentially acquired low-dose frames. This gives rise to data assimilation without significant blurring. Furthermore, it serves an important universal objective, namely to replace "human weak links" in the data processing chain by more robust, highly accurate, quantifiable, and reproducible processing modules.In this context a further important issue is to formulate and apply quantitative objective quality measures to illustrate the effectiveness of the procedures.

\section{Materials and Methods}

\subsection{Zeolites as Proxy Materials}

Zeolites are beam sensitive, crystalline aluminosilicates consisting of $\mathrm{AlO}_{4}$ and $\mathrm{SiO}_{4}$ corner-sharing tetrahedra, whose arrangement allows for a large variety of structures with different pore sizes and pore connectivities. These materials are known to deteriorate rapidly, due to a combination of radiolysis and knock-on damage [4 6], when exposed to electron beams generated by accelerating voltages between $60-200 \mathrm{kV}$.

Knock-on damage occurs when the fast electrons impart sufficient energy to the atoms in the specimen to displace them from their equilibrium positions, thereby directly breaking the long-range order of the material. $R a$ diolysis occurs when the fast electrons ionize the atoms in the material which then subsequently lose their long-range order as they seek a lower energy state. The ionization of water by the electron beam is thought to be a key step in the damage mechanism [5, 6]. Several excellent review articles on the application of transmission electron microscopy to the study of the structure of zeolites and other mesoporous materials have been recently published $7 \cdot 9]$.

Our choice of zeolites as proxies for imaging beamsensitive materials is based on four properties: (i) high crystallographic symmetry and smaller unit cell dimensions which, compared to biological objects, simplify the quantification of results and allow us to focus on technique development, (ii) the presence of water, (iii) reduced structural variety and (iv) high beam sensitivity. Each of these properties pose a significant challenge for the registration task.

\subsection{STEM Imaging}

STEM of zeolites has been used generally for either the high-contrast imaging of small metal nanoclusters within the pores using the high-angle annular dark-field (HAADF) technique or high spatial resolution chemical analysis using either energy dispersed X-ray (EDX) spectroscopy or electron energy loss spectroscopy (EELS) [10,11. Aberrationcorrected STEM allows for the formation of sub- $\AA$ probes with greatly increased brightness [12] which results in images with substantially better signal-to-noise ratio. The 

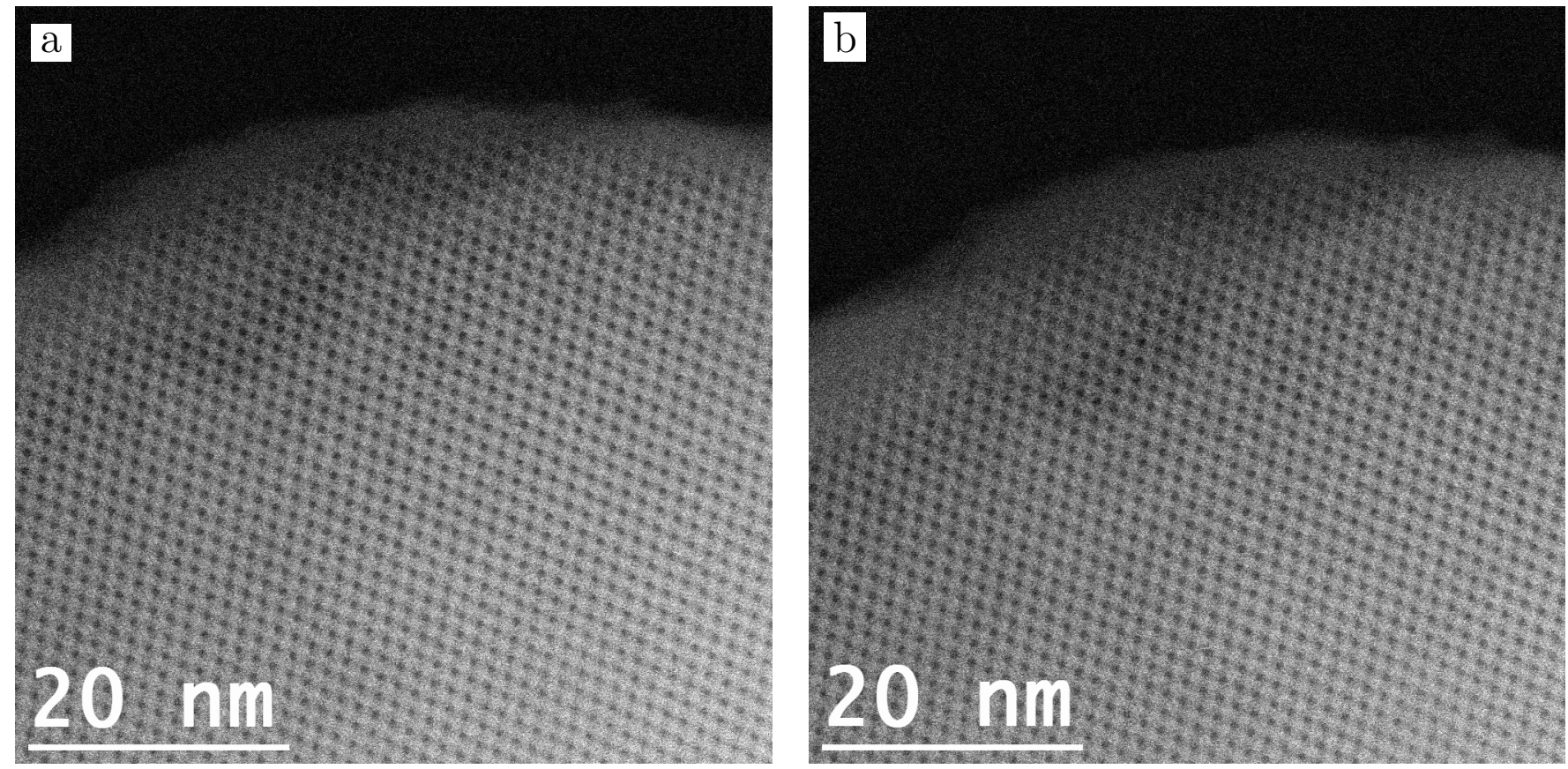

Figure 1: First (a) and ninth (b) raw frames of a HAADF STEM zeolite Si-Y series, indicating significant sample drift as well as beam damage in the region near the material's thin edge.

aberration corrector nearly eliminates the electrons in the probe outside the central maximum which results in much more dose-efficient imaging.

Ortalan et al. used an aberration-corrected STEM to image the location of single atoms of Iridium in a zeolite using a combination of low-dose image acquisition methodology and both Fourier filtering and real-space averaging [13]. The reported dose is similar to that in our work.

For our experiments HAADF STEM images at $200 \mathrm{kV}$ were recorded with a JEOL JEM 2100F TEM/STEM equipped with a CEOS CESCOR aberration corrector. All axial aberrations of the electron wave were measured and corrected up to third order. Fifth order spherical aberration was minimized as well. The illumination semi-angle was $15.5 \mathrm{mrad}$, which at $200 \mathrm{kV}$ yields a nominal probe size of $0.1 \mathrm{~nm}$. The HAADF detector recorded electrons scattered between 50 and $284 \mathrm{mrad}$. The probe current was $10 \mathrm{pA}$ as measured with a picoammeter attached to the small fluorescent focusing screen of the microscope. The pixel size was $0.3 \AA^{2}$ and dwell time per pixel was $7 \mu \mathrm{s}$ yielding an electron dose for the images of $\approx 1400 \mathrm{e}^{-} / \AA^{2}$. Image acquisition was controlled by a custom script in Digital Micrograph (Gatan, Pleasanton, CA) which sequentially recorded and saved a series of HAADF STEM images $1024 \times 1024$ in size.

Our material test sample is a siliceous zeolite Y (c.f. [14) which was completely de-aluminated. The Si-Y zeolite powder was dispersed onto an amorphous carbon holey support film on a copper mesh TEM grid. A small particle was located and oriented along the $<110>$ direction of the zeolite $\mathrm{Y}$ structure. Figure 1 shows the first and ninth frames of a series of HAADF STEM images of the Si-Y zeolite sample collected with the conditions described above. Because of the relatively low electron dose the individual images in Figure 1 are quite noisy. The largest pores of the zeolite Y structure are evident, but details of the structure are obscured by the noise level. The overall drift of the sample between the first and ninth frame is evident in the figure. For the purpose of clarifying the atomic structure of the zeolite as well as the noise level of the acquired HAADF STEM images we focus on an area $5.1 \times 7.3 \mathrm{~nm}$ in size oriented such that $<001>$ is vertical as shown in Figure 2 a. In the acquired image, the supercages of the zeolite are clear, but the atomic structure of the framework is obscured due to the noise in the image.

\subsection{Frozen Phonon Simulation of HAADF STEM image}

Figure $2 \mathrm{~b}$ shows the results of a multislice HAADF STEM image simulation using Kirkland's code [15. The simulation is equivalent to the input experimental images reported here: $200 \mathrm{kV}, 15.5 \mathrm{mrad}$ convergence semi-angle, $\mathrm{C}_{s}=3 \mu \mathrm{m}, \mathrm{C}_{5}=0 \mathrm{~mm}$ with a defocus value chosen to maximize the contrast in the image (-20 $\AA$ here). The simulation was done with a super-cell of $68.4 \AA \times 72.8 \AA$ by $120 \AA$ thick. A total of 64 phonon configurations were needed to achieve convergence of the calculation. The slice thickness was set at slightly larger than $1 \AA$. The HAADF detector spanned 50-284 mrad. Incoherent blurring was added by convolving the simulation results with the gaussian of $0.1 \mathrm{~nm}$ FWHM. Calculations were done for an area slightly larger than $1 / 4$ of the projected unit cell size of 
Si-Y $<110>$ and then appropriate symmetry was applied to generate an image $5.1 \times 7.3 \mathrm{~nm}$ in size. In this orientation the sodalite $(\beta)$ cages overlap and are not easily visualized. The largest $\alpha$-cages with a diameter of $\approx 13 \AA$ are evident and the $<001>$ direction is along the vertical direction in the Figure. The double six-member rings run between the super-cages along directions $60^{\circ}$ from the horizontal. The image simulation is effectively the ideal, infinite dose image of the perfect crystal which provides a benchmark for both the low-dose experimental images and our reconstructed image from the series.
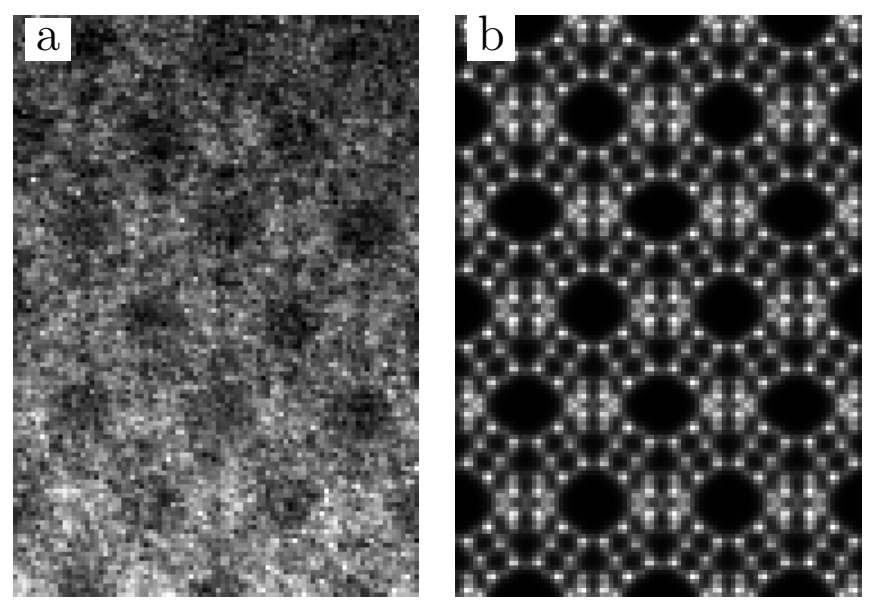

Figure 2: (a) $5.1 \times 7.3 \mathrm{~nm}$ region of the first acquired low-dose frame rotated so that $\langle 001\rangle$ is vertical. (b) Equivalent area and orientation of a HAADF STEM image simulation of the zeolite Si-Y in the $<110>$ zone axis orientation clearly revealing the large $\alpha$ cages and the double six-member rings.

\subsection{Variational Formulation of Pairwise Time Frame Reg- istration}

The usual single frame acquisition in STEM is replaced by a statistical estimate of several low-dose short-exposure frames from the same location of the specimen. By registering frames towards each other and subsequent averaging we not only achieve better results by diminishing spatial distortions but are also able to analyze beam damage and its progress over time.

\subsubsection{Nomenclature and Mathematical Notation}

The scanning procedure creates an array of intensities that we call a frame. It can be interpreted as an image reflecting the properties of the investigated specimen on some domain. However, due to distortions the locations corresponding to the measured intensities are not known exactly. Thus, the frame is not just the image but is also related to an unknown positioning system determining what exactly the image represents. The question is how the information received from several frames $f_{j}, j=1, \ldots, n$, can be effectively combined and how a specific knowledge about the distortions could be obtained.
To outline our approach we introduce some notation. It is convenient to represent a frame as a function $f$. The array values of the frame define the function values $f(x)=$ $f\left(x_{1}, x_{2}\right)$ for certain integer coordinates $x_{1}$ and $x_{2}$ on a regular, rectangular grid. Then, using an appropriate approximation method, e.g. bilinear interpolation, one can extend this function to intermediate real positions $x=$ $\left(x_{1}, x_{2}\right)$. Consider two frames $f_{j}$ and $f_{k}$. We want to establish a correspondence between their positioning systems by approximating the deformation $\phi_{j, k}$ that gives a position $\phi_{j, k}(x)$ in frame $f_{j}$ for a position $x$ in frame $f_{k}$ in the sense that $f_{j} \circ \phi_{j, k} \approx f_{k}$. The process of finding $\phi_{j, k}$ is often referred as registration of $f_{k}$ and $f_{j}$.

\subsubsection{Rigid Registration}

A particularly simple version of a registration map is a rigid motion, in general a combination of a translation and a rotation. This is typically determined by first manually aligning the respective frames, using specific image features, sometimes followed by adjustments based on numerical indicators such as the cross-correlation of the adjusted frames. In the case of STEM images, collected pixelby-pixel, rigid registration is inherently limited due to the nature of the acquisition process.

\subsubsection{Overview of Solution for Distortion Map $\phi$}

Our nonrigid series registration is founded on a variational approach that transforms two frames $f$ and $g$ into a common coordinate system with a nonparametric, nonrigid transformation $\phi$ such that $f \circ \phi \approx g$. The objective functional consists of the sum of two terms, the normalized cross-correlation of $f \circ \phi$ and $g$ as fidelity term, and the Dirichlet energy of the displacement, i. e. $\phi$ 's deviation from the identity as regularizer. While this nonparametric transformation model is very flexible, an effective numerical realization is rather difficult due to the large number of local minima our objective functional exhibits. The proposed hybrid minimization strategy rests on several important mathematical concepts:

- a multilevel scheme that processes the frames at different levels of resolution, going from coarse to fine;

- minimization on each level based on a gradient flow, a generalization of the gradient descent concept;

- an explicit time discretization of the gradient flow ODE combined with an automatic step size control to ensure convergence

\subsubsection{Nonrigid Registration}

Since feature extraction from noisy data is a challenging task by itself, we employ a registration approach that does not extract features but works directly on the image intensities. For a comprehensive introduction to image registration in general we refer the reader to the book by Modersitzki [16]. 
Ideally, assuming no noise, no distortions, and a perfect extension of the frames to real valued functions, one should expect that at any $x=\left(x_{1}, x_{2}\right)$ from the scanned domain the composition $\left(f_{j} \circ \phi_{j, k}\right)(x)=f_{j}\left(\phi_{j, k}(x)\right)$ has the same value as $f_{k}(x)$. In reality, one attempts to find a deformation $\phi_{j, k}$ that provides a good fit of $f_{j} \circ \phi_{j, k}$ to $f_{k}$ by replacing the ideal pointwise condition $\left(f_{j} \circ \phi_{j, k}\right)(x)=f_{k}(x)$ with a suitable similarity measure. Such a measure quantifies the similarity of two frames $f_{j} \circ \phi_{j, k}$ and $f_{k}$. An overview of various popular similarity measures can also be found in Modersitzki [16.

To describe such a similarity measure, as usual, we denote by $\bar{f}=\frac{1}{|\Omega|} \int_{\Omega} f \mathrm{~d} x$ the mean value of $f$ over the image domain $\Omega$. The standard deviation of $f$ over $\Omega$ is denoted by $\sigma_{f}=\sqrt{\frac{1}{|\Omega|} \int_{\Omega}(f-\bar{f})^{2} \mathrm{~d} x}$. The normalized cross-correlation of two functions $f$ and $g$ over the domain $\Omega$ is defined as

$$
\mathrm{NCC}[f, g]=\frac{1}{|\Omega|} \int_{\Omega} \frac{(f-\bar{f})}{\sigma_{f}} \frac{(g-\bar{g})}{\sigma_{g}} \mathrm{~d} x .
$$

This quantity is between -1 and 1 , while $\mathrm{NCC}[f, g]=1$ if and only if $f=a g+b$ for some real constants $a>0$ and $b$. Adopting the tradition to formulate a variational approach for determining the deformation as a minimization problem, we define the data term of our objective functional as

$$
S[\phi]=S_{f, g}[\phi]:=-\mathrm{NCC}[f \circ \phi, g] .
$$

Without further constraints, finding a deformation $\phi=$ $\left(\phi^{1}, \phi^{2}\right)$ among all vector-valued piecewise bilinear functions that minimizes $S[\phi]$ is a severely ill-posed problem. Therefore, we add the regularization term

$$
\begin{aligned}
R[\phi] & =\frac{1}{2} \int_{\Omega}\|D(\phi(x)-x)\|^{2} \mathrm{~d} x \\
& =\frac{1}{2} \int_{\Omega}\left|\frac{\partial \phi^{1}}{\partial x_{1}}-1\right|^{2}+\left|\frac{\partial \phi^{1}}{\partial x_{2}}\right|^{2}+\left|\frac{\partial \phi^{2}}{\partial x_{1}}\right|^{2}+\left|\frac{\partial \phi^{2}}{\partial x_{2}}-1\right|^{2} \mathrm{~d} x
\end{aligned}
$$

to penalize irregular displacements and receive the objective functional

$$
E[\phi]=E_{f, g}[\phi]=S_{f, g}[\phi]+\lambda R[\phi]
$$

to be minimized. The larger the regularization parameter $\lambda>0$, the smoother the resulting deformation $\phi$. This regularization term was used in Modersitzki [16] and its use is often referred to as diffusion registration.

While the non-rigid transformation model (2) is very flexible, its numerical realization is rather difficult. Due to the high beam sensitivity of zeolites, subsequent frames deteriorate rapidly and the periodic nature of typical input frames renders the estimation of the deformation $\phi$ that yields the global minimum of $E[\phi]$ particularly challenging. In fact, even after including a regularization term, the objective functional $E$ typically still exhibits a large number of local minima. Therefore, the numerical minimization requires sophisticated algorithms designed to handle a large number of local extrema. Hence, our proposed minimization strategy rests on several conceptual pillars that guide the development of the algorithmic procedures. The remainder of the current section provides the details of each component of the solution process. Primarily, it is based on a multilevel scheme that processes different levels of resolution of the data to be registered (see Subsection 2.4.5. The registration is first done on the coarsest level, the result obtained on this level is prolongated to the next finer level and used as the initialization for the minimization on this level. The process is repeated until a registration at the resolution of the input images is achieved. The minimization on each level is based on the gradient flow concept which is described in Subsections 2.4.6 and 2.4.7.

\subsubsection{Multilevel Numerical Solver for Variational Prob- lem}

A multilevel scheme serves as the outermost building block for the registration of a pair of frames. As indicated by numerous authors, e.g. [16 18] just to name a few, a coarse to fine registration strategy is a powerful tool to help prevent a registration algorithm from getting stuck in undesired local minima. The basic ideas of multilevel schemes go back to multi-grid algorithms [19, 20] and here we use the standard terminology, such as prolongation and restriction mappings, from that subject area. In particular, a hierarchy of computational grids is built, ranging from a very coarse grid level, e. g. $2^{4} \times 2^{4}=16 \times 16$ pixels, to a fine level that matches the resolution of the input images, e.g. $2^{10} \times 2^{10}=1024 \times 1024$. The exponent of 2 used to build a grid in the hierarchy is called the grid level of the multiscale grids. The registration is first done on a coarse staring level, the result obtained on this level is prolongated to the next finer level and used as the initialization for the minimization on this level. The process is repeated until a registration at the resolution of the input images is achieved. The coarser the level, the fewer structures of the input images are preserved. Hence, the minimization at a certain level avoids all local minima induced by the small structures not visible at this level. Typically grids of the size $2^{d} \times 2^{d}$ or $\left(2^{d}+1\right) \times\left(2^{d}+1\right)$ are used for multilevel schemes since there are canonical prolongation and restriction mappings to transfer data from one level to the other for these kind of grids.

In the case of $2^{d} \times 2^{d}$ grids, the grid is interpreted in a cell-centered manner. When going from one level to a finer level, each grid cell is split into two times two cells (two in each coordinate direction). The prolongation of a function copies the value from the coarse grid cell to the corresponding four grid cells in the finer grid, while the restriction uses the average value of four grid cells in the fine grid as the value for the corresponding coarse grid cell. For $\left(2^{d}+1\right) \times\left(2^{d}+1\right)$ grids a mesh-centered approach is used. Refining such a grid is done by adding a new grid node between every pair of neighboring nodes in the coarse grid. The prolongation copies the values of the 
coarse grid nodes to the nodes at the same positions in the fine grid and determines the values for the fine grid nodes that are not in the coarse grid by bilinear interpolation of the neighboring coarse grid values. The restriction is the transposition of the prolongation operation, but rescaled row-wise such that if we have a value of one on every fine grid node, we get a value of one at every coarse grid node.

\subsubsection{Gradient Flow: Analytic Formulation}

The minimization on a given level is based on a socalled gradient flow 21], a generalization of the gradient descent concept. As with the latter, the basic principle of the minimization is to go in the direction of steepest descent and formalized with the ordinary differential equation (ODE)

$$
\partial_{t} \phi=-\operatorname{grad}_{G} E[\phi] .
$$

Here, $\operatorname{grad}_{G}$ denotes the gradient with respect to a scalar product $G$. In this notation, the well-known classical gradient in finite dimensions is the gradient with respect to the Euclidean scalar product. Since a gradient flow, as a gradient descent, is attracted by the "nearest" local minimum, and the scalar product determines how distances are measured, a suitable choice of the scalar product avoids undesired local minima. As shown in [17, the ODE (3) is equivalent to

$$
\partial_{t} \phi=-A^{-1} E^{\prime}[\phi],
$$

where $E^{\prime}$ denotes the first variation of $E$ and $A$ is the bijective representation of $G$, such that $G\left(\zeta_{1}, \zeta_{2}\right)=\left\langle A \zeta_{1}, \zeta_{2}\right\rangle$ for all $\zeta_{1}, \zeta_{2}$. This formulation illustrates the influence of the scalar product and leads us to choose it such that $A^{-1}$ is smoothing its argument. This way the descent will avoid non-smooth minimizers. A Sobolev $H^{1}$ inner product scaled by $\sigma$, i.e.

$$
G_{\sigma}\left(\zeta_{1}, \zeta_{2}\right)=\int_{\Omega} \zeta_{1} \cdot \zeta_{2}+\frac{\sigma^{2}}{2} D \zeta_{1}: D \zeta_{2} \mathrm{~d} x
$$

has proved to be a suitable choice. Here, we denote $X$ : $Y=\sum_{i j} X_{i j} Y_{i j}$ for matrices $X, Y \in \mathbb{R}^{2 \times 2}$, i. e. the Euclidean scalar product of the matrices interpreted as long vectors. Note that for the corresponding metric resulting from the inner product (5), the operator $A^{-1}$ is equivalent to one implicit time step of the heat equation with a step size of $\frac{\sigma^{2}}{2}$, an operation widely known for its smoothing properties.

From (4) we see that the first variation of the objective functional $E$ (i. e. $E_{f, g}$ ) is necessary in order to use the gradient flow. With a mostly straightforward but somewhat lengthy calculation, one obtains

$$
\begin{aligned}
\left\langle E^{\prime}[\phi], \zeta\right\rangle= & -\frac{1}{|\Omega|} \int_{\Omega}(\nabla f(\phi) \cdot \zeta) \\
& {\left[\frac{(g-\bar{g})}{\sigma_{f \circ \phi} \sigma_{g}}+\frac{(f \circ \phi-\overline{f \circ \phi})}{\left(\sigma_{f \circ \phi}\right)^{2}} S[\phi]\right] \mathrm{d} x } \\
& +\lambda \int_{\Omega}(D \phi-\mathbb{1}): D \zeta \mathrm{d} x
\end{aligned}
$$

\subsubsection{Gradient Flow: Numerical discretization}

For the spatial discretization of a numerical solution to the weak form (6) we employ piecewise bilinear Finite Elements (FE) where the computational domain $\Omega$ is a uniform rectangular mesh. Introducing the FE method is beyond the scope of this paper, for a detailed introduction to the FE concept we refer to the textbook by Braess [22]. Of course, it is also possible to use different discretization approaches, for instance Finite Differences, but Finite Elements offer a canonical way to conveniently handle $\left\langle E^{\prime}[\phi], \zeta\right\rangle$, the weak formulation of the first variation of $E$. To compute the integrals in the energy, its variations and the metric, we use a numerical Gauss quadrature scheme of order 3 (see, for example, $§ 3.6$ of Stoer and Bulirsch [23]).

The gradient flow ODE (4) is solved numerically with the Euler method, an explicit time discretization approach. Thus, $\partial_{t} \phi$ is approximated by $\frac{\phi^{l+1}-\phi^{l}}{\tau}$, where $\phi^{l}$ denotes the deformation at the $l$-th iteration of the gradient flow and $\tau$ is the still-to-be-chosen step size. This leads to the update formula

$$
\phi^{l+1}=\phi^{l}-\tau A^{-1} E^{\prime}\left[\phi^{l}\right] .
$$

Since the minimization does not require the knowledge of the trajectory of $\phi$ given by the ODE but only the equilibrium point, it is sufficient to use this kind of simple explicit method to solve the ODE. It is very important though to carefully choose the step size $\tau$ since the iteration can diverge with a poorly chosen step size. To this end we employ the so-called Armijo rule with widening [24]. This rule is a line search method used on the function

$$
\Phi(\tau)=E\left[\phi^{l}-\tau A^{-1} E^{\prime}\left[\phi^{l}\right]\right]
$$

and selects the step size such that the ratio of the secant slope $\frac{\Phi(\tau)-\Phi(0)}{\tau}$ and the tangent slope $\Phi^{\prime}(\tau)$ always exceeds a fixed, positive threshold $\rho \in(0,1)$. This means that the actual achieved energy decay (secant slope) is at least $\rho$ times the expected energy decay (tangent slope). Note that the specific choice of the step size control is not important - there are several popular step size controls that could be used here. It is crucial though to use a step size control that guarantees the convergence of the time discrete gradient flow (7).

The resulting multilevel descent algorithm is summarized in Algorithm 1 .

Our multilevel descent algorithm is well-suited for the beam sensitive materials we consider here. Beam damage starts as a loss of high frequency information in the image as the atomic structure of the sample is lost with increasing electron irradiation. At the coarser stages of the registration, we find the distortion map for the large scale differences between the image frames. As the registration proceeds, the distortions for the earlier distortion map are used as the initial values for the next level. In the case where either the noise level in the input frames is 


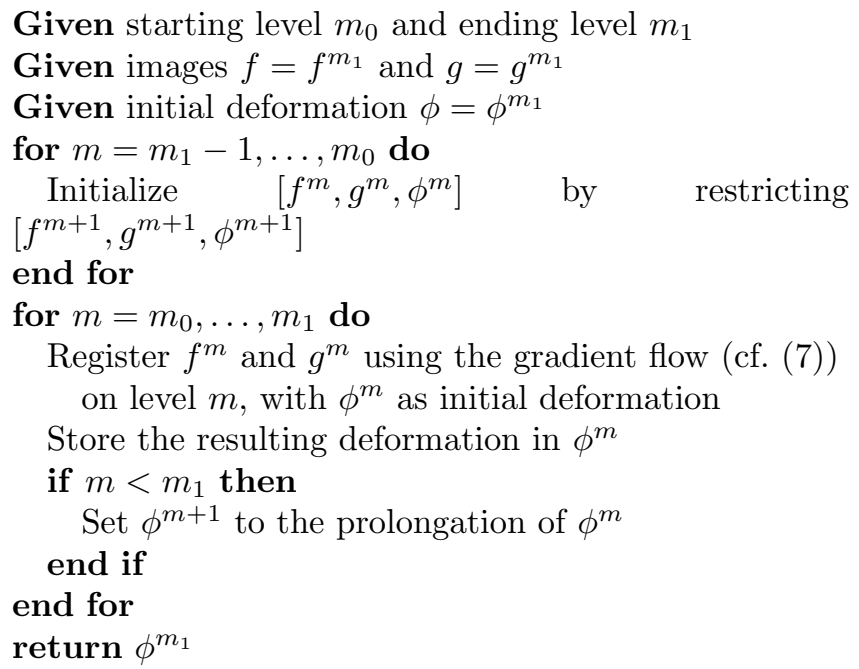

\section{ALGORITHM 1: Multilevel gradient flow}

extremely high or the material has changed from frame to frame, the distortions from the coarser level are retained and the regularization of the algorithm smoothly adjusts between the coarser grid points. The step size control described above means that our registration proceeds only to the point where the comparison between the frames is still meaningful. When either the noise level of the input or a significant difference in the two frames occurs, the registration algorithm is terminated, minimizing the artifacts of our method.

\subsection{Registration of a frame series}

Now that we have proposed how to register a pair of images, we still have to provide a concept on how to register a series of images when the goal is to fuse the information contained in all images of the series into a single image. The basic idea here is to register all of the input frames to a common reference frame. Once this is achieved one can simply calculate a suitable average of the registered images pixel by pixel to get the reconstructed image.

A natural strategy here is to simply select one of the images as reference frame and then to use the registration method for image pairs to register all of the other images to the selected image. Figure 1 indicates some of the potential pitfalls of this strategy by showing two frames of a series that are several frames apart. The left image depicts the first frame of the series, the right image depicts the ninth frame. Most strikingly, the specimen moved considerably between the two frames. Moreover, the thin edge region of the specimen appears differently in both images. The material is so beam sensitive that the scanning already caused visible damage to part of the specimen in the latter image. In combination with the periodic structure of the material these effects increase the difficulty when registering this image pair. A good initial guess for the registration where the spatial error is already less than half of the unit cell size of the specimen should be sufficient to overcome these problems. In order to obtain such an accurate initialization we can exploit properties of the acquisition process of the series (see Subsection 2.5.1 for details).

The main procedure for registering a series is summarized by the steps

- use of the identity as an initial guess only when registering consecutive frames and subsequently employ the composition of the already established transformations for this purpose, since the specimen can move considerably during the series' acquisition;

- register the series to a single reference frame (usually the first) and calculate a suitable pixel-wise average of the registered series to fuse the information contained in all frames into a single one;

- iterate this procedure with lowered weight on the regularizer using the average as new common reference frame.

which are fully described in the following subsections.

\subsubsection{Basic Strategy for a Series}

Since consecutive images in our input series were acquired directly one after another, we can assume the differences between two consecutive images, both regarding displacement and beam damage, to be small enough to use the identity as initialization for the minimization on the coarsest level in Algorithm 1. In case this assumption is not fulfilled it may be necessary to add a fiducial mark to the specimen to facilitate the registration algorithm.

After having a suitable deformation for each consecutive image pair we can chain those to get estimates for non-consecutive pairs. Let $f_{1}, \ldots f_{n}$ denote the images in our series ordered by their time of acquisition. As outlined above we can estimate deformations $\phi_{2,1}$ and $\phi_{3,2}$ that match the first and second image pair respectively, i. e. $f_{2} \circ \phi_{2,1} \approx f_{1}$ and $f_{3} \circ \phi_{3,2} \approx f_{2}$. Then $\phi_{3,2} \circ \phi_{2,1}$ is a good initial guess for the deformation matching $f_{1}$ and $f_{3}$ because $f_{3} \circ\left(\phi_{3,2} \circ \phi_{2,1}\right)=\left(f_{3} \circ \phi_{3,2}\right) \circ \phi_{2,1} \approx f_{2} \circ \phi_{2,1} \approx$ $f_{1}$. Therefore, our proposed registration algorithm with $\phi_{3,2} \circ \phi_{2,1}$ as initial guess allows us to reliably register $f_{3}$ to $f_{1}$. Iterating this provides a way to accurately register any frame of the series to the first frame (cf. Figure 3 ).

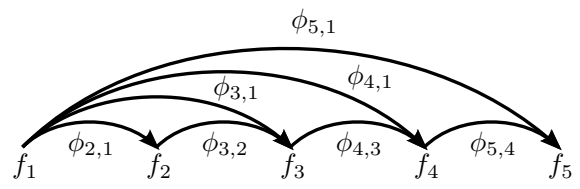

Figure 3: Strategy to match a series of consecutive images.

After registering all frames to the first one, we can fuse the information contained in the series by a suitable averaging operator $\mathcal{A}$ acting on the deformed frames, $g_{i}=f_{i} \circ \phi_{i, 1}$, where $\phi_{1,1}$ is the identity mapping. Given a 
sequence of frames $\left\{g_{i}\right\}_{i=1}^{n}$ the first example is the mean

$$
\mathcal{A}\left[\left\{g_{i}\right\}_{i}\right](x)=\frac{1}{n} \sum_{i=1}^{n} g_{i}(x)
$$

which provides the best least squares fit to the sequence. The arithmetic mean is a natural choice to calculate an average and is very easy to compute, although, depending on the input data, it may not be the best choice. In particular, if the input data contains noticeable outliers, which due to the scattering nature of the electrons in the HAADF STEM measurements is expected to be the case, the median is preferable to the arithmetic mean. Recall the definition of the median

$$
\mathcal{A}\left[\left\{g_{i}\right\}_{i}\right](x) \in \underset{g \in \mathbb{R}}{\operatorname{argmin}} \sum_{i=1}^{n}\left|g_{i}(x)-g\right|,
$$

which is the best $\ell_{1}$ fit to the sequence and therefore less sensitive to outliers. This selection of $\mathcal{A}$ is used in all subsequent experiments.

\subsubsection{Iteration of Basic Strategy}

The estimated reconstructed image $f=\mathcal{A}\left\{f_{i} \circ \phi_{i, 1}\right\}$ from the previous section can be considerably improved by iteration of this basic procedure. Choosing $f_{1}$ as reference frame was a canonical but arbitrary choice, whereas the newly obtained average $f$ is a much more suitable reference frame. Thus, it is reasonable to repeat the process using $f$ as reference frame. Since $f_{1}$ was the reference frame when calculating $f$ the identity mapping is a good initialization for the registration of $f_{1}$ to $f$ and we can simply prepend $f$ to the input series, calling it $f_{0}$, and repeat the process. Here however, we don't include $f_{0}$ when calculating the average since the original images $f_{1}, \ldots f_{n}$ obviously contain all of our measurement information. Thus, an average of the deformed frames may be calculated via $f=\mathcal{A}\left[\left\{f_{i} \circ \phi_{i, 0}\right\}_{i}\right]$. This results in a further improved reconstructed image $f$ and thus the process can be repeated again, leading to an iteration procedure to calculate $f$.

The $k$-th estimate of $f$ is denoted by $f^{k}$. Initially, $f^{0}=f_{1}$ since the specimen is least damaged during the acquisition of $f_{1}$. We use $f_{0}=f^{k-1}$ as our reference frame at this stage and calculate $f^{k}$ using $\mathcal{A}\left[\left\{f_{i} \circ \phi_{i, 0}^{k}\right\}_{i}\right]$ where $\phi_{i, 0}^{k}$ denotes the deformation from $f_{i}$ to $f_{0}=f^{k-1}$ at the end of each iteration.

A critical issue in finding the deformations $\phi_{i, 0}^{k}$ is how to determine the initial guess for the nonrigid registration process we have described. Since the specimen can move considerably during the acquisition of the image series, we use the identity as an initial guess only for the transforms $\phi_{j+1, j}$ between two consecutive frames (note that $\phi_{1,0}^{0}$ is the identity). Then the initial guess for $\phi_{1,0}^{k+1}$ is the transform $\phi_{1,0}^{k}$, while for $\phi_{j+1,0}^{k}$ it is the composition $\phi_{j+1, j}^{k} \circ \phi_{j, 0}^{k}$. Note that the estimation of $\phi_{j+1,0}^{k}$ depends on $\phi_{j, 0}^{k}$, so as indicated before, these deformations need to be determined one after another. As an example, in Figure 4 we present frame 9 registered to frame 1.

This core methodology opens an avenue to further algorithmic options which facilitate an even increased denoising effectiveness at the expense of additional computational effort (see Subsection 3.5.2. The point is that within some regime, in principle, additional computational work gains increased information quality. The full strategy is summarized in Algorithm 2

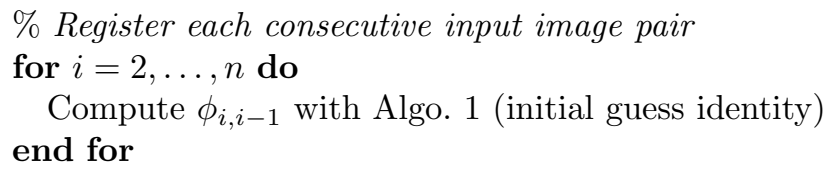

Initialize 0 -th average with $f^{0}:=f_{1}$

for $k=1, \ldots, K$ do

Select last average as reference frame, i. e. $f_{0}:=f^{k-1}$

$\%$ Registration part

Compute $\phi_{1,0}^{k}$ with Algo. 1 (initial guess identity)

for $i=2, \ldots, n$ do $\left.\phi_{i-1,0}^{k}\right)$

Compute $\phi_{i, 0}^{k}$ with Algo. 1 (initial guess $\phi_{i, i-1}$ 。

end for

\section{$\%$ Averaging part \\ Obtain average $f^{k}$ pixel-wise via $(8)$ or end for}

\section{ALGORITHM 2: Series averaging procedure}

Note that after the first time the average has been computed, i. e. when $k \geq 2$, the reference frame $f_{0}$ is considerably less noisy than the input data. Thus, it is then possible to reduce the regularization parameter $\lambda$ when calculating $\phi_{i, 0}^{k}$ for $k \geq 2$. Here, we typically use $0.1 \lambda$ as regularization parameter for $k \geq 2$, i. e. the same regularization parameter value is used for all $k \geq 2$, but this value is smaller than the one used for $k=1$.

\section{Result and Discussion}

An application of the algorithm to our test series of nine frames produces the image b) in the Figure 5 below. For comparison, a manual, rigid alignment of the same input frames was performed and median averaging applied. The resulting image is shown in Figure 5 a. The non-rigid algorithm required slightly over 2 hours of computation on an Intel i5-3335s@2.7GHz for an average time to register two $1024 \times 1024$ frames of 3.66 minutes.

\subsection{Metric for Success of Reconstruction.}

Figure 6] shows a magnified portion of Figure 5 for both the manual rigid registration as well as the algorithm described above. The reference area for the manual alignment, near the material thin edge, is included in this magnified segment, which represents the best-case results of 

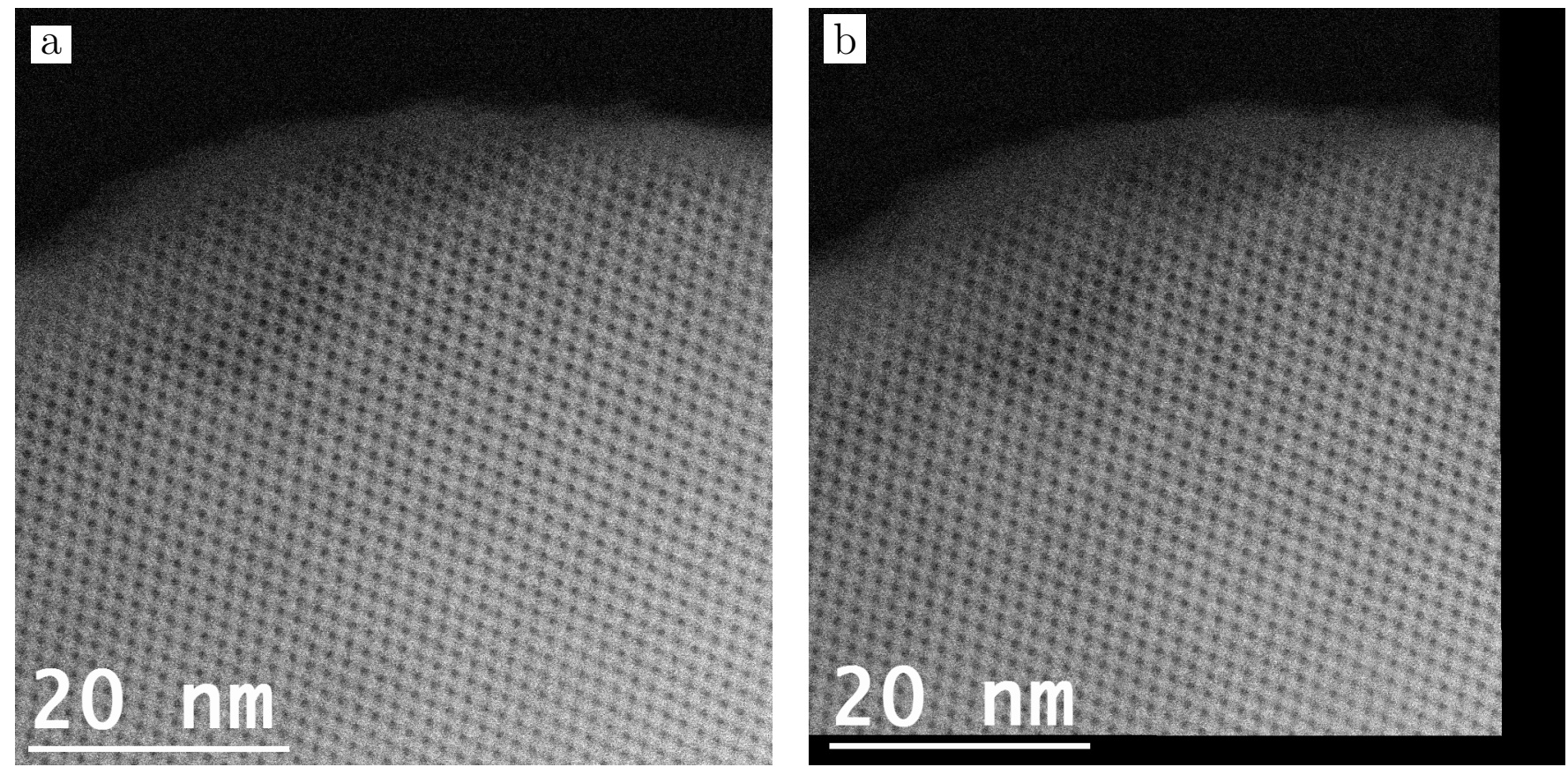

Figure 4: Registration of the raw data. The raw data of the first frame of a HAADF STEM image series (a) and the ninth frame (b) following the non-rigid registration procedure. The solid black at the bottom and right edge of the registered image are parts of the field of view from the first frame, and used in the reconstruction from the full image series, which do not appear in the ninth frame.
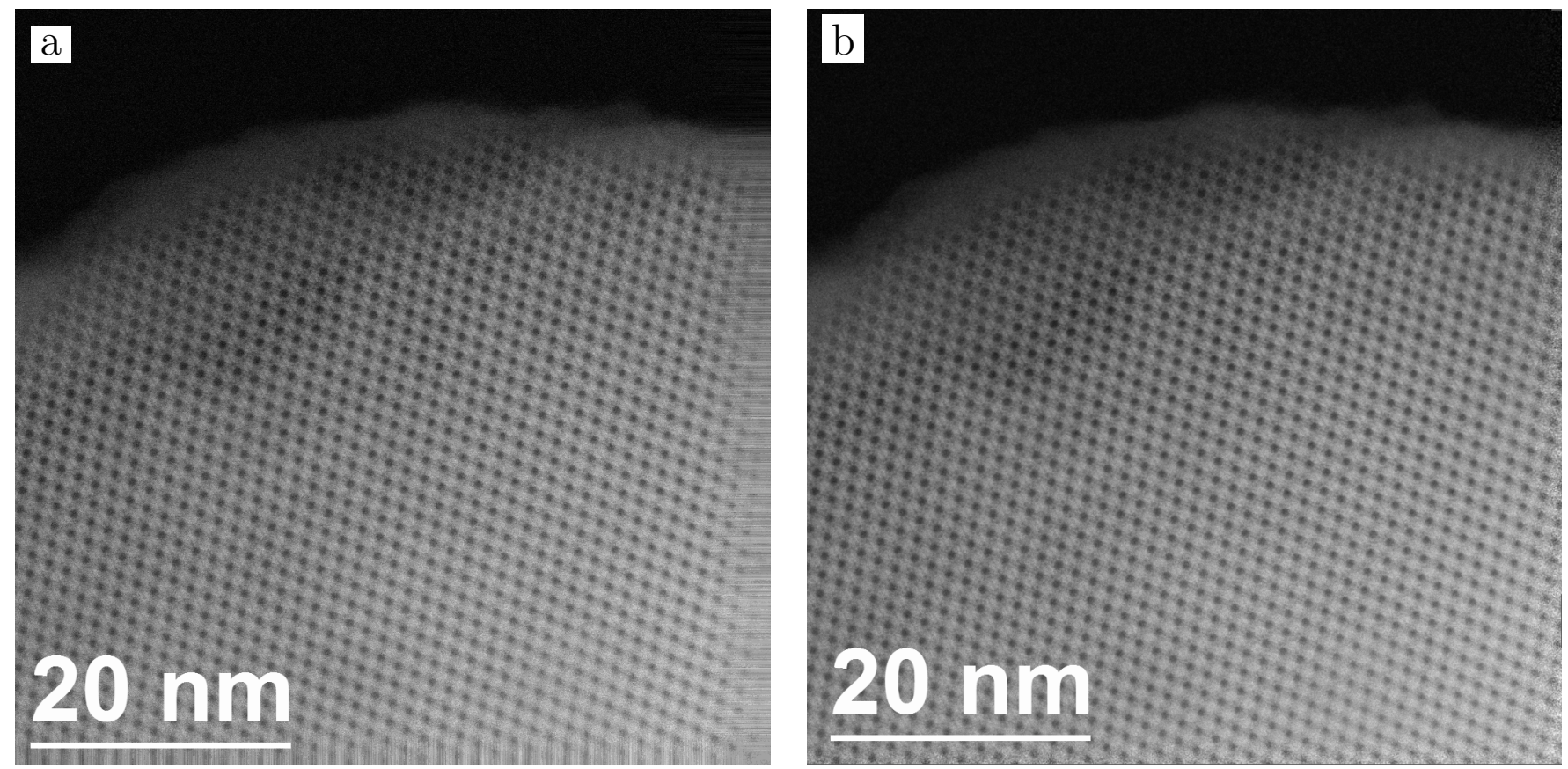

Figure 5: High resolution, full frame results of manual and nonrigid registration. Resulting average images produced by registration of 9 frames for a) manual and b)nonrigid registration, followed by median averaging. (Images are embedded in the on-line PDF at full resolution.) 

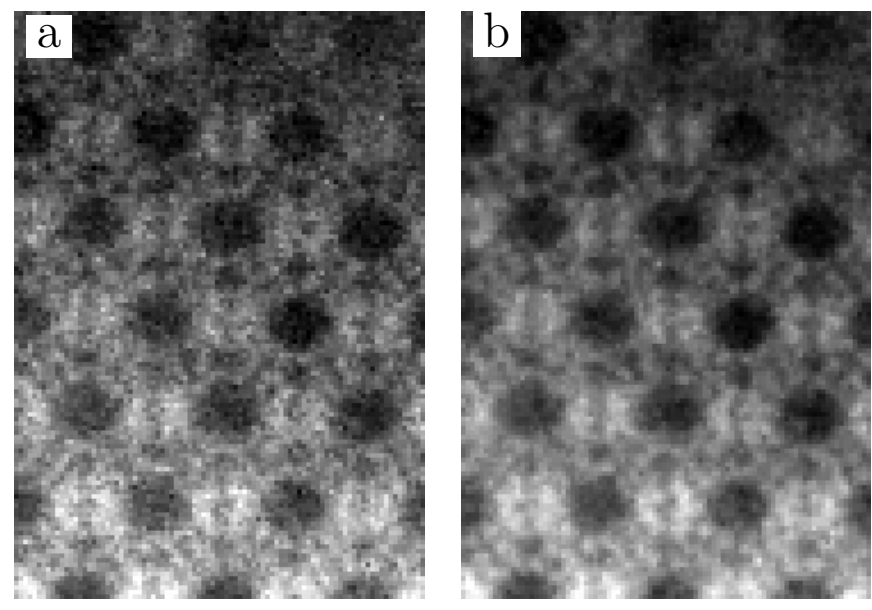

Figure 6: Results of frame assimilation. Segments of the assembled images produced by the two methods of registration of 9 frames, followed by median averaging. The displayed region is equivalent to that of the input frame seen in Figure 2 a) Resulting image segment from manual rigid registration of 9 image frames. b) An image segment produced with the aid of a non-rigid registration of the 9 image frames. Super-cages and the double six-member rings are resolved across this entire segment, as they are in the full image seen in Figure 5

the rigid alignment. The image in Figure 6b exemplifies the successful implementation of the described paradigm for a series of nine HAADF STEM images of the Si-Y zeolite sample. In particular, this illustrates the effect of the algorithm for beam sensitive materials. The improvement offered by the computational procedure is visually apparent as even the secondary pores become visible essentially over the complete image area covered by the material. Figure 1 shows the substantial translation undergone by the specimen during the acquisition process. How to factor out such a translation from the series of frames is outlined in the Section 3.5.1 which could further improve the registration quality.

\subsection{Quantitative Measures of Quality}

As important as a complete robust and accurate information processing chain is a quantitative quality assessment that can serve as a basis for subsequent scientific conclusions. The following discussion should be viewed as a step in this direction. One aspect is to concede that perhaps a "single number" as "measure of quality" can hardly serve our purpose. In view of the complexity of the information carried by the data, as the comparison of rigid versus nonrigid registration shows, one should rather incorporate localizing measures, examples of which are suggested below.

In order to quantify the quality of the registration, we consider one type of information content remaining in the quantity

$$
d=f_{n}-f_{0} \circ \phi_{0, n},
$$

i. e. the difference between the last experimentally acquired frame $f_{n}$ and our average $f_{0}$ nonrigidly transformed back to $f_{n}$. Since the algorithm we have described in the previous subsection calculates $\phi_{n, 0}$ instead of $\phi_{0, n}$, at first glance it might seem natural to consider the difference $f_{0}-f_{n} \circ \phi_{n, 0}$. However, this comparison would be flawed because the experimentally acquired data $f_{n}$ would then have been manipulated before being compared to the calculated reconstruction. To calculate $\phi_{0, n}$ we simply register our average $f_{0}$ to $f_{n}$ with Algorithm 1, where a numerical inverse of the already calculated $\phi_{n, 0}$ is used as an initial guess. Note that to prevent any smoothing in the pixel-wise calculation of $f_{0} \circ \phi_{0, n}, f_{0}$ is evaluated at the deformed pixel positions using nearest neighbor interpolation, not bilinearly like in the FE-based registration algorithm.

In case $f_{0}$ is the noiseless, undistorted image we aim to reconstruct and $\phi_{0, n}$ perfectly captures the distortion of the target image $f_{0}$ to the $n$-th measured frame $f_{n}, d$ contains nothing but noise. Thus, the higher the quality of the registration and averaging procedure, the closer $d$ is to pure noise. Moreover, in order to compare different methods one can consider a measurement that evaluates the amount of information in $d$. Of course, in our particular example where the frames change due to accumulation of beam damage during the acquisition $d$ will contain the induced changes between the frames. Since the induced change is primarily the loss of high frequency information, it will be difficult to distinguish between the high frequency noise in the frames due to the low-dose nature of the acquisition.

We define such measurements in the next subsection. Figure 7 summarizes the performance of the manual and the nonrigid registration methods based on these procedures. Figure $7 \mathrm{a}$ is $d$ for the manual, rigid registration, while Figure $7 \mathrm{p}$ is $d$ for the non-rigid registration. For the manual rigid alignment, a small portion of the image consists of noise, while the majority of the frame contains significant structural information which will be blurred upon averaging the frames. The non-rigid registration approaches the ideal of pure noise throughout the entire frame.

\subsection{IQ Factor for estimating Image Quality.}

In the case that $d$ is identical to noise, the power spectrum of $d$ should contain no maxima in its modulus. Evaluating the power spectrum of $d$ will then provide a measurement of the quality of the registration. The IQ-factor introduced by Unwin and Henderson 25 provides a well known method of measuring the local maxima in the modulus of a power spectrum of an image. The IQ-factor is the ratio of the maximum of the modulus of a spot in the power spectrum to the average local background in the power spectrum. In this case, since we are interested in measuring the error in the registration process by quantifying how close $d$ is to pure noise, a low IQ indicates excellent registration while a high IQ-factor indicates failure in the registration process. Note that this is inverse to the usual meaning of the IQ-factor. Figure $7 \mathrm{c}$ shows how 

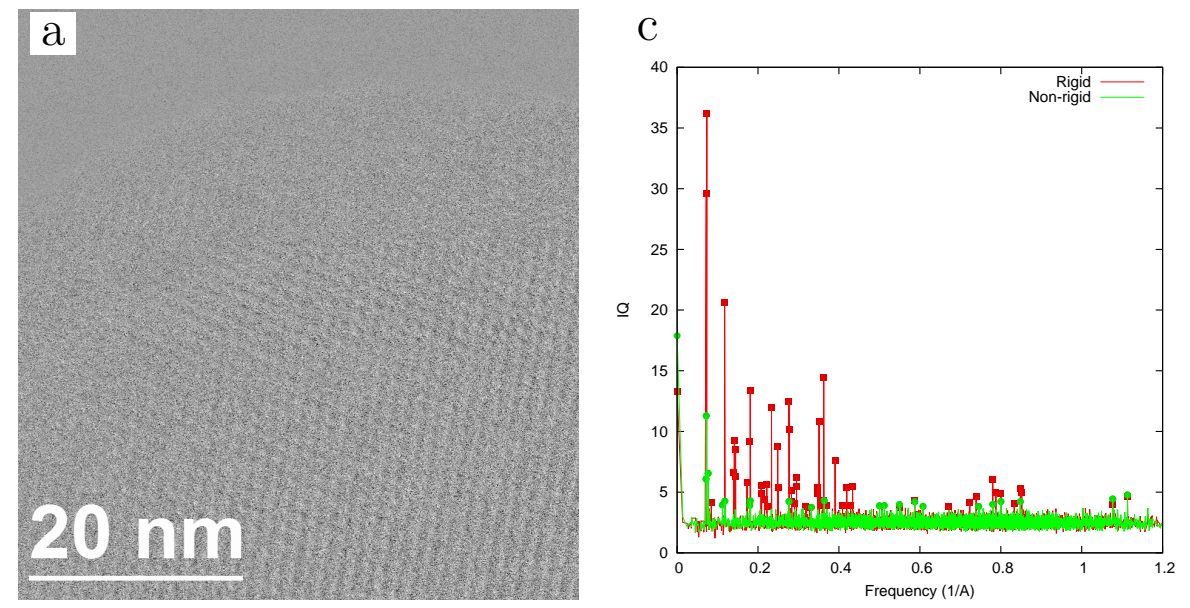

e
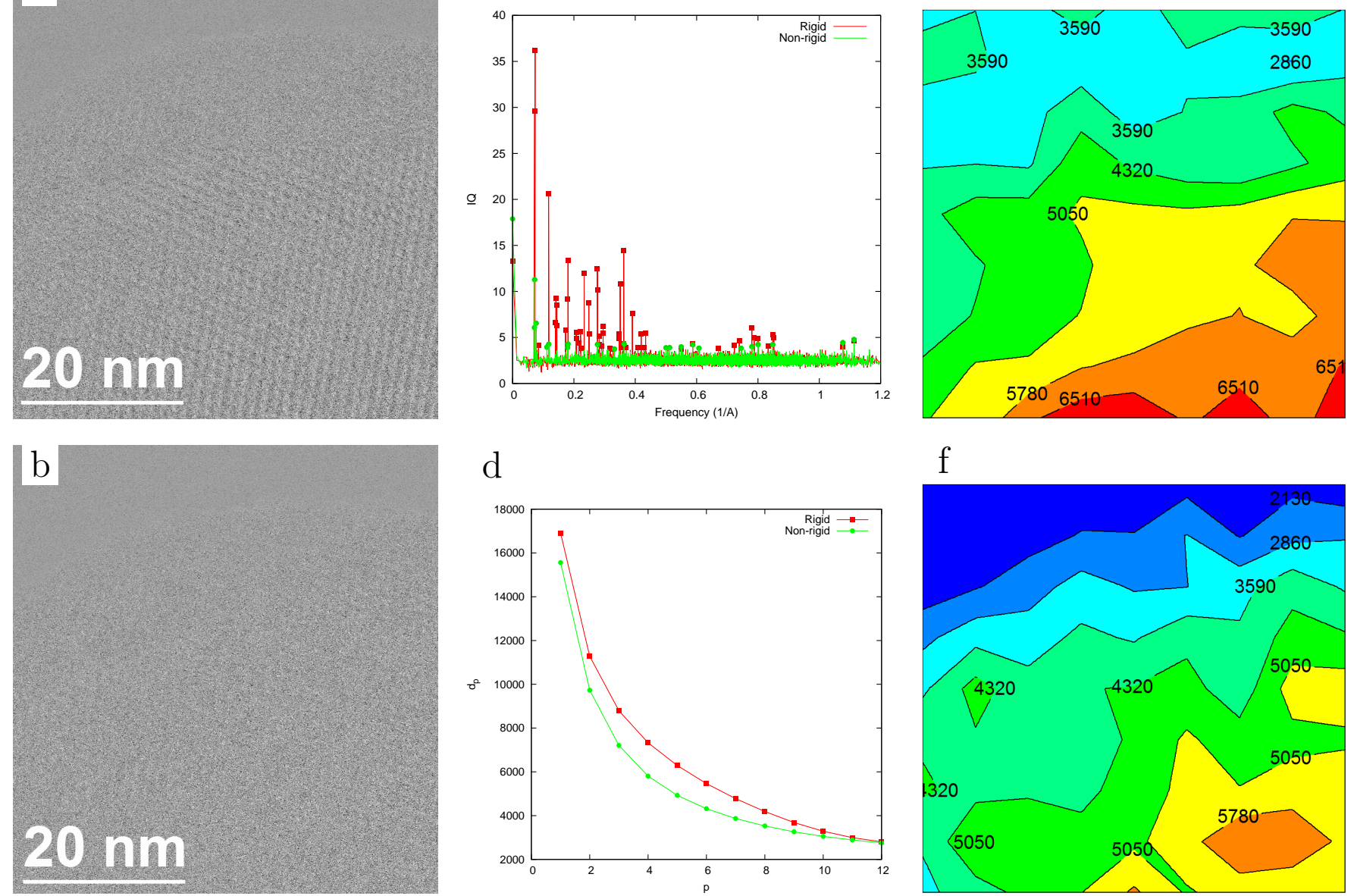

Figure 7: Quality of Reconstructions. The left column of images in the figure show the difference $d=f_{0} \circ \phi_{0,9}-f_{9}$ evaluated at the deformed pixel positions using nearest neighbor interpolation, a) for the manual rigid registration and $\mathbf{b}$ ) for the automatic non-rigid registration, cropping as needed. In the case of perfect alignment one would expect only noise in these images. c) IQ plots of images a) (red) and b) (green) plotted against distance from the origin. Smaller IQ values indicate smaller residual information in an image. d) Arithmetic mean of $d_{p}$ (as the patch radius $p=1, \ldots 12$ varies) for the manual rigid (red) and the automatic non-rigid registration (green) of the first nine frames of a HAADF STEM time series, where the median was used to calculate the average image. e) Arithmetic mean of $d_{2}$ for $9 \times 9$ subregions for the manual rigid registration. f) Arithmetric mean of $d_{2}$ for $9 \times 9$ subregions for the automatic non-rigid registration.

$d$ for the manual rigid alignment of the HAADF STEM image series (Figure 7 a) contains a significant amount of non-noise information with a maximum IQ of more than 35. In contrast, the $d$ of our non-rigid registration (Figure 7 b) has only a few spatial frequencies with residual signal other than noise.

\subsection{Quantifying the Quality for Registration of a Series.}

Another potential metric for the quality of the registration would be the absolute value of the average of $d$ in a small region. Let $p \in \mathbb{N}$. To evaluate how close $d$ is to noise, we average $d$ over all patches of size $(2 p+1) \times(2 p+1)$ and consider the absolute value of the average, resulting in an image $d_{p}$ of size $(N-2 p) \times(N-2 p)$, i. e.

$$
d_{p}(\boldsymbol{i})=\left|\frac{1}{(2 p+1)^{2}} \sum_{\boldsymbol{k} \in P_{p}^{i_{1}+p, i_{2}+p}} d(\boldsymbol{k})\right| .
$$

Since the averaging over the patches reduces the noise in $d$, the higher the quality of the registration and averaging procedure, the smaller the values of $d_{p}$. Thus, the arithmetic mean of $d_{p}$ gives a quantitative measure of the quality of the procedure, the lower the better. In particular, this quantity allows for the comparison of the quality of different registration results. Figure $7 \mathrm{~d}$ shows how $d_{p}$ for the non-rigid registration is always smaller than $d_{p}$ for the manual registration as $\mathrm{p}$ varies from 1 to 12 .

To get a more local view of the quality measure, we can partition $d_{p}$ in $L \times L$ patches of equal size (dropping pixels close to the boundary if the width or height of $d_{p}$ is not a multiple of $L$ ) and calculate the arithmetic mean in these patches instead of the whole image. Figure 7 is a contour plot of $d_{2}$ on a $9 \times 9$ grid for the manual registration, while Figure $7 \mathrm{f}$ is the same for the non-rigid registration. Note that the manual registration always performed more poorly than the non-rigid registration even in the area used 
as the reference for the manual registration.

The lower right hand portion of the reconstructions (Figure 5) correspond to an area of the initial field of view where the sample was not in focus due to a thickness gradient in the particular specimen. Our algorithm correctly registered this area of the frames on a coarse scale, but did not artificially "improve" the image in this area. The reconstruction varies in quality across the field of view as the input frames vary in information. This is a distinct advantage over some other image processing techniques which either enforce periodicity on the images, or produce an "average" structure.

\subsection{Further Algorithm Extensions}

The above procedure should be viewed as one realization of a general concept that in principle allows one to extract additional information at the expense of additional computation. We briefly highlight two such possibilities. The first deals with single frame adjustments to the acquisition process. The second addresses an iterative extension of the above algorithm for a series of frames.

\subsubsection{A first order motion extraction.}

The inevitable movement of the sample during the scanning process combined with how STEM rasters the electron probe on the sample to acquire an image leads to a spatial distortion in the STEM images. In particular, Figure 4 shows a significant translation of the field of view encountered during the acquisition of the series of frames. In order to retain as much information as possible and ease the registration process by minimizing frame-to-frame distortions we introduce next a simple strategy for capturing the most prominent "macroscale" translational parts of the distortion. To formulate this model we need to make assumptions on the movement of the sample and need to denote some quantities inherent to the STEM scanning process.

Let $t$ denote the time it takes to scan a single line in the image and let $t_{f}$ denote the flyback time, i. e. the amount of time it takes to move the STEM probe from the end of one line to the beginning of the next line. As first order approximation, we assume that the sample undergoes a constant translational movement $v=\left(v_{1}, v_{2}\right) \in \mathbb{R}^{2}$ while a STEM image is acquired. The movement vector may differ from frame to frame though. Furthermore, we assume that the STEM probe starts to scan the sample at position $(0,0)$. Thus, when we scan the first pixel, corresponding to the position $(0,0)$, we also scan position $(0,0)$ of the sample. When scanning the last pixel in the first line, corresponding to the position $(1,0)$, the time $t$, i. e. the time to scan a line, has passed and the sample has moved by $t v$. Hence, we are actually scanning the position $(1,0)-t v$ of the sample. Let $h$ denote the height of a STEM line as fraction of 1 , i. e. $h=\frac{1}{N-1}$ where $N$ denotes the number of lines in the STEM image. Then, when scanning the first pixel of the second line, corresponding to the position
$(0, h)$, the time $t+t_{f}$ has passed and we are actually scanning the position $(0, h)-\left(t+t_{f}\right) v$. Consequently, when scanning the last pixel of the second line, corresponding to the position $(1, h)$, the time $2 t+t_{f}$ has passed and we are actually scanning the position $(1, h)-\left(2 t+t_{f}\right) v$.

In general, when scanning a position $x_{1} \in[0,1]$ in line $k$, corresponding to $\left(x_{1}, k h\right)$, a time of $t x_{1}+\left(t+t_{f}\right) k$ has passed and we are actually scanning the position $\left(x_{1}, k h\right)-$ $\left(t x_{1}+\left(t+t_{f}\right) k\right) v$. Therefore, assuming that the distortion is linearly interpolated between the lines, the image domain is deformed by the linear mapping

$$
x \mapsto\left(\begin{array}{cc}
1-t v_{1} & -\frac{\left(t+t_{f}\right) v_{1}}{h} \\
-t v_{2} & 1-\frac{\left(t+t_{f}\right) v_{2}}{h}
\end{array}\right) x .
$$

In other words, denoting the matrix by $M$, instead of $f(x)$ the intensity acquired at position $x$ is actually showing $f(M x)$. Thus, using the inverse of $M$, it would be possible to remove the distortion caused by a constant translation of the sample during the acquisition of the frame.

\subsubsection{An extended series averaging procedure.}

Algorithm 2 allows us to generate a reconstruction of the series that structurally closely resembles the first frame $f_{1}$ due to the fact that $f_{1}$ was used as initial reference frame in the algorithm. In particular, if we drop the outer loop, i. e. for $K=1$, the reconstruction actually is a denoised version of $f_{1}$ (note that this is not guaranteed for $K \geq 2$ ). Using a slight alteration of Algorithm 2, we can register all frames to the $j$-th frame. This is achieved by applying the algorithm with $K=1$ and, without the averaging step, once on the series $f_{j-1}, \ldots, f_{1}$ and once on the series $f_{j+1}, \ldots, f_{n}$, each time using $f_{j}$ as reference frame. Having the transformations of all frames to the $j$ th frame, we can create a denoised reconstruction $\tilde{f}_{j}$ of the $j$-th frame and thus of every input frame. Furthermore, we can use the algorithm to register all of the denoised frames to the first of these frames $\tilde{f}_{1}$. Contrary to the initial registration of the input frames to $f_{1}$, the registration of the denoised frames can be done with a considerably lower regularization parameter $\lambda$ and at a higher precision since this registration is not hampered by the large amount of noise found in the input frames. Due to their improved accuracy, the deformations determined based on $\tilde{f}_{1} \ldots, \tilde{f}_{n}$ can be used to generate an improved reconstruction from our original input $f_{1}, \ldots, f_{n}$ by using these deformations when averaging the input frames with $f_{1}$ as reference frame. If desired, this process can even be iterated, i. e. instead of only generating an improved reconstruction of $f_{1}$ one can generate an improved reconstruction of every frame and again create an improved average of the original data by registering the improved reconstructions and so on, which can be seen as an extension of the $k$-loop idea in Algorithm 2. Let us emphasize that all reconstructions here are always obtained by averaging the original noisy input frames, only the deformations used for the averaging were 
determined based on reconstructions we calculated previously.

The main drawback of this variant is the vastly increased computational cost compared to our original algorithm. Since the original algorithm essentially needs to be run $n+1$ times, once for each input frame to get a denoised version of this frame and once to create the improved reconstruction based on the registration of the denoised frames, the computational cost is increased by a factor of $n+1$. Thus, it is only feasible to use this approach if the computational cost is not a concern or if the number $n$ of input frames is moderate.

\section{Conclusions}

We have developed a strategy for extracting an increased level of information from a series of low dose STEM images, rather than using single high dose images, in order to circumvent or at least significantly ameliorate a build up of unwanted physical artifacts, contortions, and damage caused during the acquisition process. We have applied the methodology to beam sensitive materials like siliceous zeolite Y.

A crucial ingredient is a non-linear registration process that removes visual inspection and human interaction. The quantitatively improved automated information retrieval, is an important step forward since the huge amounts of data created by many modern imaging techniques employed in astrophysics, medical imaging, process control and various forms of microscopy call for an early and reliable triage before storage. In particular, in many of these areas change detection is crucial and critically hinges on an accurate and reliable registration.

We provide algorithms and metrics that allow researchers to extract larger amounts of meaningful information from data which often are costly to acquire. The quality of the final reconstruction hinges on the quality of the initial input data. In the case considered here of a beam sensitive zeolite under low-dose conditions, significant improvement in the information content of the reconstruction was demonstrated relative to the individual input frames without artificially "improving" the areas in the field of view which either were not in focus in the input frames or suffered significant beam damage during the series acquisition.

\section{Acknowledgements}

This research was supported in part by the MURI/ARO Grant W911NF-07-1-0185; the NSF Grants DMS-0915104 and DMS-1222390; the Special Priority Program SPP 1324, funded by DFG; and National Academies Keck Futures Initiative grant NAKFI IS11.

\section{References}

[1] P. Binev, F. Blanco-Silva, D. Blom, W. Dahmen, P. Lamby, R. Sharpley, and T. Vogt. High-Quality Image Formation by Nonlocal Means Applied to High-Angle Annular Dark-Field Scanning Transmission Electron Microscopy (HAADFSTEM). In T. Vogt, W. Dahmen, and P. Binev (eds.), Modeling Nanoscale Imaging in Electron Microscopy, Nanostructure Science and Technology, pp. 127-145, (Springer US2012).

[2] R. Leary, P. A. Midgley, and J. M. Thomas. Recent Advances in the Application of Electron Tomography to Materials Chemistry. Acc. Chem. Res. (2012).

[3] S. Nickell, F. Frster, A. Linaroudis, W. D. Net, F. Beck, R. Hegerl, W. Baumeister, and J. M. Plitzko. TOM software toolbox: acquisition and analysis for electron tomography. Journal of Structural Biology 149(3), 227 (2005).

[4] O. Ugurlu, J. Haus, A. A. Gunawan, M. G. Thomas, S. Maheshwari, M. Tsapatsis, and K. A. Mkhoyan. Radiolysis to knock-on damage transition in zeolites under electron beam irradiation. Phys. Rev. B 83(113408) (2011).

[5] M. M. J. Treacy and J. M. Newsam. Electron beam sensitivity of zeolite L. Ultramicroscopy 23, 411 (1987).

[6] L. A. Bursill, E. A. Lodge, and J. M. Thomas. Zeolitic structures as revealed by high-resolution electron microscopy. Nature $\mathbf{2 8 6}$, 111 (1980).

[7] M. Pan. High Resolution Electron Microscopy of Zeolites. Micron 27(3-4), 219 (1996).

[8] J. M. Thomas, O. Terasaki, P. Gai, W. Zhou, and J. GonzalezCalbet. Structural Elucidation of Microporous and Mesoporous Catalysts and Molecular Sieves by High-Resolution Electron Microscopy. Acc. Chem. Res. 34, 583 (2001).

[9] I. Diaz and A. Mayoral. TEM Studies of zeolites and ordered mesoporous materials. Micron 42, 512 (2011).

[10] D. Ozkaya, W. Zhou, J. M. Thomas, P. Midgeley, V. J. Keast, and S. Hermans. High-resolution imaging of nanoparticle bimetallic catalysts supported on mesoporous silica. Catalysis Letters 60, 113 (1999).

[11] J. M. Thomas, T. G. Sparrow, M. K. Uppal, and B. G. Williams. Chemical analyses and studies of electronic-structure of solids by electron-energy loss spectroscopy with an electron microscope. Philos. Trans. R. Soc. London Ser. A 318, 259 (1984).

[12] O. L. Krivanek, N. Delby, and A. R. Lupini. Towards sub- $\AA$ electron beams. Ultramicroscopy 78, 1 (1999).

[13] V. Ortalan, A. Uzan, B. C. Gates, and N. D. Browning. Direct imaging of single metal atoms and clusters in the pores of dealuminated HY zeolite. Nature Nanotechnology 5, 506 (2010).

[14] J. A. Hriljac, M. M. Eddy, A. K. Cheetham, J. A. Donohue, and G. J. Ray. Powder Neutron Diffraction and ${ }^{29}$ Si MAS NMR Studies of Siliceous Zeolite-Y. Journal of Solid State Chemistry 106(1), 66 (1993).

[15] E. J. Kirkland. Advanced Computing in Electron Microscopy, (Plenum, New York1998).

[16] J. Modersitzki. Numerical Methods for Image Registration, (Oxford University Press2004).

[17] U. Clarenz, M. Droske, and M. Rumpf. Towards fast non-rigid registration. In Inverse Problems, Image Analysis and Medical Imaging, AMS Special Session Interaction of Inverse Problems and Image Analysis, vol. 313, pp. 67-84, (AMS2002).

[18] J. Han, B. Berkels, M. Droske, J. Hornegger, M. Rumpf, C. Schaller, J. Scorzin, and H. Urbach. Mumford-Shah Model for One-to-One Edge Matching. IEEE Transactions on Image Processing 16(11), 2720 (2007).

[19] W. Briggs. A Multigrid Tutorial, (Society for Industrial and Applied Mathematics (SIAM), Philadelphia 1987).

[20] W. Hackbusch. Multigrid Methods and Applications, (Springer Series in Computational Mathematics, 4. Springer-Verlag, Berlin1985).

[21] G. Sundaramoorthi, A. Yezzi, and A. Mennucci. Sobolev Active Contours. International Journal of Computer Vision. 73(3), 345 (2007).

[22] D. Braess. Finite Elements, (Cambridge University Press2007), 3rd ed. 
[23] J. Stoer and R. Bulirsch. Introduction to numerical analysis, vol. 12 of Texts in Applied Mathematics, (Springer, New York2002), 3rd ed. Translated from the German by R. Bartels, W. Gautschi and C. Witzgall.

[24] L. Armijo. Minimization of functions having Lipschitz continuous first partial derivatives. Pacific Journal of Mathematics 16(1), 1 (1966).

[25] P. Unwin and R. Henderson. Molecular Structure Determination by Electron Microscopy of Unstained Crystalline Specimens. Journal of Molecular Biology 94, 425 (1975). 